\title{
ABOLITION OF ASTIGMATISM OF OBLIQUE BUNDLES IN CASE OF A SINGLE LENS
}

BY

James P. C. Southale

\section{So-Called "Punktal" Sfectacle Lens}

The determination of the form and dimensions of a single lens which, for a prescribed refracting power $(F)$ and for a given position of the object, shall be free from astigmatism of oblique bundles of rays, is an optical problem of considerable practical importance for a number of purposes; on which therefore much has been written, particularly of recent years in connection with the investigation of the most satisfactory forms of modern spectacle lenses. Among those who have made notable contributions to this special phase of the problem may be mentioned Tscherning, Percival, Ostwalt, von Rohr and Gullstrand. Perhaps now more than ten years ago, the Zeiss Optical Company placed on the market a form of spectacle'lens called the "Punktal" calculated and designed by von Rohr; which is also made in this country by the Bausch \& Lomb Optical Company.

The curvatures and thickness of the "Punktal" lens depend on the power of the glass, which means that it is expensive to manufacture and consequently has not yet come into very extensive use. There is no particular theoretical difficulty about designing a lens of this type; in fact the necessary formulae have long been known. The chief difficulty in the way of its practical realization consisted perhaps in recognizing the essential fact that the centre of rotation of the eye (and not, for example, the centre of the pupil) was the point of reference in this particular optical combination which was the effective point for the determination of the chief rays of the oblique bundles which it was the business of the lens to render stigmatic before they entered the eye.

For positive lenses of powers higher than between 7 and 8 dioptries, the theory shows that no form of "Punktal" lens is 
possible; and for such higher powers of positive spectacle lenses as are needed, for example, in cases of very high hypermetropia or of aphakia (when the eye has been operated on for cataract), Gullstrand has designed a lens with an aspherical surface which is made by Zeiss under the name of "Katral" lens. As heretofore comparatively little has been published in English about the so-called "Punktal" lens (or about similar types of lenses made by other manufacturers), the following notes may be of interest, especially if they lead to criticism and discussion on the part of such workers in the optical research laboratories as have been engaged recently in studying the properties of this important type of spectacle lens.

In ordinary direct vision the eye is mobile and turns almost involuntarily to look at the point of fixation which is sharply focused on the retina in the fovea centralis. The lines of fixation (which show the direction in which the eye is gazing) all pass through a fixed point called the eye-pivot or centre of rotation of the eye (designated always hereafter by $Z^{\prime}$ ) which may be assumed to lie on the optical axis of the eye at a distance of $13 \mathrm{~mm}$ beyond the vertex of the anterior surface of the cornea. In general, therefore, the optical axis of a mobile ametropic eye corrected by a spectacle glass will not coincide with the optical axis of the correction-glass; and consequently a spectacle glass which may be all right when used centrally will usually show astigmatism when the eye turns in its socket to look obliquely through the lens. In order to see distinctly through the glass, no matter how the eye turns behind it, the form of the lens must be specially designed so that the astigmatism of oblique bundles of rays is abolished. Practically, the problem amounts to computing the form of the lens for the case of a small rear stop placed beyond it with its centre at the place where the centre of rotation of the eye would be when the lens is in use in its right position in front of the eye; so that within a certain field of fixation corresponding to the angular movement of the eye-ball the astigmatism of oblique cylindrical bundles of incident rays will be abolished by the lens and delivered to the eye as if they emanated, really or virtually, from a point lying approximately at least on the so-called "far point sphere" of the mobile eye. 
The distance between the eye and the nearer surface of a correction-glass (measured from the vertex of this surface to the vertex of the anterior surface of the cornea) should ordinarily be not less than about $12 \mathrm{~mm}$. Thus, if the vertices of the two surfaces of the lens are designated by $A_{1}$ and $A_{2}$, then the distance $\mathrm{A}_{2} \mathrm{Z}^{\prime}$ should generally be not less than about $25 \mathrm{~mm}$; although it may be as great as $30 \mathrm{~mm}$. However, within these limits, a smaller value of this distance is usually better for two reasons, first, because the astigmatism of a lens with a rear stop is more easily corrected in this case, and, second, because the nearer the centre of rotation of the eye is to the correction-glass, the smaller the diameter of the lens will have to be for a given angular excursion of the eyeball; which means that the spectacles may be made both smaller and lighter, as is always desirable.

\section{Notation}

The form of the required lens (that is the curvatures $R_{1}, R_{2}$ of its two surfaces and its axial thickness $d=\mathrm{A}_{1} \mathrm{~A}_{2}$ ) will depend on its refracting power $(F)$, the place of the object, the distance of the stop, and of course also the index of refraction of the glass (n). If

$$
F_{1}=(\mathrm{n}-1) R_{1}, \quad F_{2}=-(\mathrm{n}-1) R_{2},
$$

denote the refracting powers of the two surfaces of the lens, then

$$
F=F_{1}+F_{2}-c F_{1} F_{2}
$$

where $c=d / \mathrm{n}$ denotes the "reduced" thickness of the lens. If the principal points of the lens are designated by $\mathrm{H}, \mathrm{H}^{\prime}$, then $\mathrm{A}_{1} \mathrm{H}=$ $c F_{2} / F$ and $\mathrm{A}_{2} \mathrm{H}=-c F_{1} / F$. Moreover, let $z, z^{\prime}$ designate the positions on the axis of another pair of conjugate points, one of which is to be regarded as the centre of the small stop. If the lens is a spectacle correction-glass for an ametropic eye, the point $Z^{\prime}$ (as already explained) must be chosen to coincide with the centre of rotation of the eye, and in this case the calculation must be made, therefore, for a lens with a rear stop with its centre at $Z^{\prime}$. Now if we put $z=1 / Z=\mathrm{HZ}, z^{\prime}=1 / Z^{\prime}=\mathrm{H}^{\prime} Z^{\prime}$, then $Z^{\prime}=Z+F$. The distance of the object from the lens may be denoted by $u_{1}=1 / U_{1}$; and since it will be assumed that the object is at an infinite distance (as is always the case for a so-called correction-glass in spectacle optics), we shall have $U_{1}=0$. 
From a point of the object which is not on the axis there will proceed a narrow cylindrical bundle of incident rays, the chief ray being the one which is directed towards the point $Z$. After passing through the lens this chief ray will cross the axis at a point $Z^{\prime \prime}$ which will usually be not far from the point $Z^{\prime}$ and which would actually coincide with $Z^{\prime}$ if the lens were free from spherical aberration with respect to the pair of conjugate axial points $Z$, $Z^{\prime}$. After emerging from the lens the narrow bundle of rays will generally be astigmatic, that is, the so-called meridian (or tangential) rays will intersect ("really" or "virtually") at a point $\mathrm{P}^{\prime}$ on the chief ray, whereas the sagittal rays will focus at another point $\mathrm{Q}^{\prime}$ on this same ray. The interval $\mathrm{P}^{\prime} \mathrm{Q}$ ' is called the "astigmatic difference" of the bundle; and in the special case when $Q^{\prime}$ coincides with $\mathrm{P}^{\prime}$, so that the astigmatic difference vanishes, the bundle of emergent rays will be stigmatic or monocentric; and the lens may be said to give a punctual image of the object. This is what is meant here by abolishing the astigmatism.

\section{Case of an Infinitely Thin Lens}

As is always the case with a problem of this kind, the investigation will be greatly simplified provided it is permissible to neglect entirely the thickness of the lens; that is, to regard the points designated above by $A_{1}, A_{2}$, and $H, H^{\prime}$ as all coincident at the optical centre of the lens. In a spectacle lens this simplifying assumption is not very far from correct, although it is less permissible in modern meniscus forms of lenses than in the older forms of spectacle lenses. Now according to Seidel's famous approximate expressions for the spherical aberrations of an optical system, ${ }^{1}$ the condition of the abolition of the astigmatism of oblique bundles of rays for an infinitely thin lens and an infinitely distant object may be expressed by an homogeneous equation of the 3rd degree in which the variables are the magnitudes denoted above by the symbols $F, F_{1}$, and $Z^{\prime}$ and the coefficients are functions of the index of refraction (n). This equation may be written as a quadratic in $F_{1}$, as follows:

$$
\text { a } F_{1}{ }^{2}+\beta F_{1}+\gamma=0, \quad\left(d=0, U_{1}=0\right) ;
$$

${ }^{1}$ See, for example, Southall's Mirrors, Prisms and Lenses (The Macmillan Company, New York, 1918), Article 193. 
where

$$
\begin{gathered}
a=\frac{\mathrm{n}+2}{\mathrm{n}(\mathrm{n}-1)^{2}} F, \quad \beta=-\left\{a F+\frac{2(\mathrm{n}+1)}{\mathrm{n}(\mathrm{n}-1)} Z^{\prime}\right\} F, \\
\gamma=\left(\frac{F}{\mathrm{n}-1}+Z^{\prime}\right)^{2} F .
\end{gathered}
$$

Accordingly, for given values of $F$ and $Z^{\prime}$, two values of $F_{1}$ may be found which will satisfy this equation, namely:

$$
F_{1}=\frac{(\mathrm{n}+2) F+2\left(\mathrm{n}^{2}-1\right) Z^{\prime} \pm \sqrt{(\mathrm{n}+2)(2-3 \mathrm{n}) F^{2}-4(\mathrm{n}-1)^{2}(\mathrm{n}+2) F Z^{\prime}+4(\mathrm{n}-1)^{2} Z^{\prime 2}}}{2(\mathrm{n}+2)} .
$$

The refracting power of the other surface will be $F_{2}=F-F_{1}$. In the case of a spectacle correction-glass, the actual values of $F_{1}$ are found to be positive always and those of $F_{2}$ negative; so that the form of the required lens (whether convex or concave) is of the meniscus type (except in one special case mentioned below, when the lens is planoconcave).

For a given value of $Z^{\prime}$ (for example, when $z^{\prime}=25 \mathrm{~mm}$ or $Z^{\prime}$ $=40$ dioptries) the quadratic equation with variables $F$ and $F_{1}$, referred to a system of rectangular axes with these variables as coördinates, will be found to represent an ellipse which is tangent to the $F$-axis (abscissa-axis) at the point $F=-(\mathrm{n}-1) Z^{\prime}, F_{1}=0$. The form of lens corresponding to this special point on the curve is therefore planoconcave. Except at this point, the ellipse lies wholly above the abscissa-axis, and hence (as was stated above, assuming that $Z^{\prime}>0$, as it must be for the case of a rear stop), the form of the lens is invariably that of a meniscus. The coordinates of the centre of the ellipse are:

$$
-\frac{2(\mathrm{n}-1)^{2}}{3 \mathrm{n}-2} Z^{\prime}, \quad+\frac{2 \mathrm{n}^{2}}{(\mathrm{n}+2)(3 \mathrm{n}-2)} Z^{\prime}
$$

and the squares of its semi-axes, major and minor, will be found by dividing $2 \mathrm{n}^{3}(\mathrm{n}-1)^{2} Z^{\prime}$ by

$$
(n+2)(3 n-2)\left\{n+(n+2)(n-1)^{2} \pm \sqrt{n^{2}+(n-1)^{2}(n+2)\left(n^{3}-4 n+4\right)}\right\} .
$$

Thus, for a given value of $Z^{\prime}$, there may always be found two real values of $F_{1}$ corresponding to each value of $F$ comprised between the limits of the two extreme values of $F$, namely:

$$
F=\frac{2(\mathrm{n}-1)}{3 \mathrm{n}-2}\left\{-(\mathrm{n}-1) \pm \mathrm{n} \sqrt{\frac{\mathrm{n}}{\mathrm{n}+2}}\right\} Z^{\prime}
$$

the upper sign corresponding to the highest possible positive 
value of $F$ and the lower sign to the extreme negative value. For example, for $\mathrm{n}=1.52$, the two extreme values are $F=+0.19452 Z^{\prime}$ and $F=-0.6170 Z^{\prime}$; which shows that the range on the negative side extends more than three times as far as on the positive side. Putting $Z^{\prime}=40 \mathrm{dptr}$, the limiting values of $F$ are found to be $+7.78 \mathrm{dptr}$. and $-24.68 \mathrm{dptr}$. It may happen that the required power of the correction-glass is greater than this extreme positive value, as was stated above; and in such a case no form of so-called "Punktal" spectacle lens is to be had, and a "Katral" lens with an aspherical surface will have to be employed. On the other hand, the extreme negative value of $F$ is more than sufficient to satisfy the requirements of a correction-glass with punctual imagery for the worst cases of myopia.

As to the two solutions of the quadratic equation in $F_{1}$, one of them gives a meniscus lens of shallower form than the other, and it is this shallower type that corresponds to the modern "Punktal" spectacle lens. The other more concave form is similar to the so-called "periscopic" eye-glass which was recommended by Dr. Wollaston more than a century ago.

If the magnitude denoted by $Z^{\prime}$ is regarded as a variable parameter, the equation $a F_{1}^{2}+\beta F_{1}+\gamma=0$ will represent a family of ellipses of similar character, as has been pointed out by $\mathrm{H}$. Boegehold in a paper published in 1920 in Zs. f. ophthalm. Optik, 8, p. 10-16. These ellipses all have a pair of common tangents which intersect at the origin of the rectangular system of coördinates. One of these tangents (as mentioned already) is the abscissaaxis itself which touches each ellipse at the point for which $F=-(\mathrm{n}-1) Z^{\prime}$, corresponding always to a comparatively high negative value of $F$. The equation of the other common tangent to this family of ellipses is $F_{1}=\mathrm{n}^{2} F$. This straight line touches each ellipse at a point whose coördinates are:

$$
F=\frac{\mathrm{n}-1}{\mathrm{n}(\mathrm{n}+1)-1} Z^{\prime}, \quad F_{1}=\mathrm{n}^{2} F .
$$

\section{General Case; with Numerical Examples}

If, however, the thickness of the lens is taken into account, Seidel's expression for the condition that a single lens of unit 
refracting power $(F=1)$, of refractive index $\mathrm{n}$ and of thickness $d=\mathrm{n} c$, shall be free from astigmatism of oblique bundles for the case of an infinitely distant object, leads to the following formula arranged here as an equation of the 5 th degree in $F_{1}$ :

$$
\begin{aligned}
& c^{3} F_{1}{ }^{5}-\left\{\mathrm{n} c(\mathrm{n}+2)-2 c S_{1}+1\right\} c F_{1}^{4} \\
&+\left\{c S_{1}^{2}-2\left(\mathrm{n}^{2} c+\mathrm{n} c+1\right) S_{1}+\mathrm{n}\left(2 \mathrm{n}^{2} c+\mathrm{n} c+2 \mathrm{n}+4\right)\right\} c F_{1}^{3} \\
&-\left\{\left(\mathrm{n}^{2} c+2 \mathrm{n} c-\mathrm{n}^{2}+1\right) c S_{1}^{2}+2 \mathrm{n}\left(\mathrm{n}^{2}-2 \mathrm{n}^{2} c-\mathrm{n} c-\mathrm{n}-3\right) c S_{1}\right. \\
&+\left.+\mathrm{n}\left(\mathrm{n}^{3} c^{2}+4 \mathrm{n}^{2} c+2 \mathrm{n} c+\mathrm{n}+2\right)\right\} F_{1}^{2} \\
&- \mathrm{n}\left(2 \mathrm{n}^{2}-2 \mathrm{n}^{2} c-\mathrm{n} c-2\right) c S_{1}^{2}+2 \mathrm{n}\left(\mathrm{n}^{3} c^{2}-\mathrm{n}^{3} c+3 \mathrm{n}^{2} c+\mathrm{n} c-\mathrm{n}^{2}+1\right) S_{1} \\
&-\mathrm{n}^{2}\left\{\left(\mathrm{n}^{3} c^{2}-2 \mathrm{n}^{2} c+2 \mathrm{n} c+\mathrm{n}^{2}-2 \mathrm{n}+1\right) \mathrm{n}^{2}\left(2 \mathrm{n}^{2} c+2 \mathrm{n}+1\right)\right\} F_{1} \\
&\left.-2 \mathrm{n}(\mathrm{n} c-\mathrm{n}+1) S_{1}+\mathrm{n}^{2}\right\}=0, \quad\left(F=1, U_{1}=0\right) .
\end{aligned}
$$

In this equation $S_{1}=1 / s_{1}$ where $s_{1}=\mathrm{A}_{1} \mathrm{Z}$ denotes the distance of the point designated by $Z$ from the first surface of the lens. The equation above does not contain the magnitude $F$, because it applies to the case of a lens of unit power. In order to obtain the corresponding solution for a lens of any power $F$, positive or negative; all the linear magnitudes (denoted by the small italic letters) must be divided, and all the reciprocal magnitudes (denoted by the capital italic letters) must be multiplied, by the given value of $F$.

This equation may be arranged also either as a quadratic in $S_{1}$, namely:

$$
\begin{aligned}
& \left\{\left(F_{1}{ }^{3}-X\right) c^{2}+(\mathrm{n}-1)\left(\mathrm{n}^{3}-\mathrm{n} F_{1}-F_{1}{ }^{2}+\frac{X}{\mathrm{n}}\right) c-\mathrm{n}^{2}(\mathrm{n}-1)^{2}\right\} S_{1}{ }^{2} \\
& \quad+2\left\{\left(F_{1}{ }^{3}-X+\mathrm{n} F_{1}{ }^{2}\right) c^{2} F_{1}-\left(F_{1}{ }^{3}-X+Y F_{1}\right) c+Y\right\} S_{1} \\
& \quad-X\left(1-c F_{1}\right)-c(1-c)\left(F_{1}^{3}-X\right) F_{1}=0, \quad\left(F=1, U_{1}=0\right),
\end{aligned}
$$

or as a quadratic in $c$, namely:

$$
\begin{aligned}
& \left\{\left(F_{1}^{3}-X\right)\left(S_{1}+F_{1}\right)^{2}+2 \mathrm{n} S_{1} F_{1}^{3}\right\} c^{2} \\
& \quad-\left\{(\mathrm{n}-1)\left(F_{1}^{2}+\mathrm{n} F_{1}-\mathrm{n}^{3}-\frac{X}{\mathrm{n}}\right) S_{1}{ }^{2}+2\left(F_{1}^{3}-X+Y F_{1}\right) S_{1}+F_{1}\left(F_{1}^{3}-2 X\right)\right\} c \\
& \quad-\mathrm{n}^{2}(\mathrm{n}-1){ }^{2} S_{1}{ }^{2}+2 Y S_{1}-X=0,\left(F=1, U_{1}=0\right) ;
\end{aligned}
$$

where the symbols $X$ and $Y$ are employed as abbreviations for the following expressions:

$$
\begin{aligned}
& X=\mathrm{n}\left\{(\mathrm{n}+2) F_{1}^{2}-\mathrm{n}(2 \mathrm{n}+1) F_{1}+\mathrm{n}^{3}\right\}, \\
& Y=\mathrm{n}(\mathrm{n}-1)\left\{(\mathrm{n}+1) F_{1}-\mathrm{n}^{2}\right\} .
\end{aligned}
$$

Equation (4) implies that if a lens is given completely (that is, if the magnitudes denoted by $\mathrm{n}, c$ and $F_{1}$ are known and $F=1$ ), 
two positions of the narrow diaphragm may be computed for which the optical system will be free from astigmatism of oblique bundles for an infinitely distant object.

Similarly, for a known value of $\mathbf{n}$ and for a given curvature of the first surface of the lens and for a fixed position of the stop with respect to this surface, equation (5) implies that there will be two values of the thickness $(d=n c)$ of a lens of unit power which will satisfy the condition of freedom from astigmatism of oblique bundles for an infinitely distant object.

If the absolute term of the quadratic in $c$, equation (5) above, is put equal to zero, the equation obtained is equivalent to the condition given by equation (2) for the case of an infinitely thin lens of unit power.

(1) A numerical example will illustrate better than anything else the method of using these formulae. Suppose that it is required to determine the form of a spectacle-lens of refracting power $F=4$ dptr., made of glass of index $\mathrm{n}=1.52$, which shall be free from astigmatism of oblique bundles for a prescribed distance of the centre of rotation of the eye from the correctionglass. This latter distance $\mathrm{A}_{2} \mathrm{Z}^{\prime}$; as : we saw, must lie between 25 and $30 \mathrm{~mm}$, but the difficulty here consists in the fact that until the form of the lens has been ascertained, it is impossible to assign precisely the position on the axis of the corresponding point designated by $\mathrm{Z}$; because what we really need to know is the value of $s_{1}=\mathrm{A} Z$ in order to substitute in equation (3) the value of $S_{1}=$ $1 / s_{1}$. If we assume that $z^{\prime}=\mathrm{H}^{\prime} Z^{\prime}=30 \mathrm{~mm}$, that is, $Z^{\prime}=1 / z^{\prime}=$ $331 / 3$ dptr., then, since $Z=Z^{\prime}-F$, we shall have $Z=291 / 3$ dptr. Now if we put $S_{1}$ equal to this value of $Z$, we shall obtain finally a value of $\mathrm{A}_{2} \mathrm{Z}^{\prime}$ which for a suitable lens-thickness will be comprised between the limiting values 25 and $30 \mathrm{~mm}$. It remains only to choose the value of the thickness of the lens which we shall take here as $3 \mathrm{~mm}(d=\mathrm{n} c=0.003 \mathrm{~m})$. All these values are for a lens of refracting power $4 \mathrm{dptr}$. Hence, before substitution in equation (3) the value of $c$ above must be multiplied, and that of $S_{1}$ divided, by 4 ; thus, we obtain for substitution: $\mathrm{n}=1.52$, $S_{1}=71 / 3$ and $\log c=7.8973377-10$. Evidently, the numerical 
work is rather long and laborious. If equation (3) is written in the form

$$
F_{1}^{5}+A F_{1}^{4}+B F_{1}^{3}+C F_{1}^{2}+D F_{1}+E=0,
$$

we find finally with the above data:

$$
\begin{aligned}
& \log A=2.1334844-, \quad \ldots \quad \log B=2.6949651-, \quad \log C=4.8645063-, \\
& \log D=5.7627989+, \quad \log E=6.0085579-.
\end{aligned}
$$

The cöefficients $A, B, C, D$ and $E$ having been determined, it remains now to ascertain the one root of this equation of the 5 th degree which is applicable for our purposes. Under ordinary circumstances this would be a tedious task; but luckily it is comparatively easy to find an approximate value of $F_{1}$ which will enable us to locate the exact value without much difficulty. For this purpose all we have to do is to substitute in equation (2) the values $F=1, Z^{\prime}=(331 / 3) / 4=81 / 3 \mathrm{dptr}$; ; whence we obtain for the shallower type of meniscus lens the approximate value: $F_{1}$ $=2.78$ dptr. $(F=1, d=0)$. With this clue to aid us, it is now a comparatively simple matter to ascertain the root of equation (3) which we are trying to find and which turns out to be: .

$$
F_{1}=2.74162 \text { dptr., }(F=1 \text { dptr.). }
$$

Accordingly, for a lens of power 4 dptr., we obtain finally the following system of values:

$$
\begin{aligned}
& F=+4.00000 \text { dptr., } \mathrm{n}=1.52, d=3.0000 \mathrm{~mm}, \mathrm{~A}_{1} \mathrm{Z}=+34.0909 \mathrm{~mm} ; \\
& F_{1}=+10.96648 \mathrm{dptr}, F_{2}=-7.10423 \mathrm{dptr} ; \\
& \text { Radii: } r_{1}=+47.4172 \mathrm{~mm}, r_{2}=+73.1959 \mathrm{~mm} ; \\
& \mathrm{A}_{1} \mathrm{H}=-3.5054 \mathrm{~mm}, \mathrm{~A}_{2} \mathrm{H}^{\prime}=-5.4111 \mathrm{~mm} \text {; } \mathrm{A}_{2} \mathrm{Z}^{\prime}=+27.2704 \mathrm{~mm} .
\end{aligned}
$$

The field of fixation of the mobile eye has an angular extent of from $50^{\circ}$ to $60^{\circ}$, say. Consider a narrow cylindrical bundle of incident rays inclined to the axis of the lens at an angle of $28^{\circ}$ as representing the outermost bundle. The chief ray directed towards the point $Z$ emerges from the lens and crosses the axis at a point $Z^{\prime \prime}$ such that $Z^{\prime \prime} Z^{\prime}=+0.78 \mathrm{~mm}$; and the astigmatic difference of this outside bundle of rays as found by exact trigonometric computation is $\mathrm{P}^{\prime} \mathrm{Q}^{\prime}=-3.35 \mathrm{~mm}$.

The results of similar calculations by means of these formulae are given below for several other cases.

(2) For the following arbitralily' assigned values, namely:

$$
F=+4.250 \text { dptr., } \mathrm{n}=1.52, d=3.5294 \mathrm{~mm}, \mathrm{~A}_{1} Z=+33.6129 \mathrm{~mm} \text {; }
$$


the computation gives:

$$
\begin{aligned}
& F_{1}=+11.3645 \mathrm{dptr}, \quad F_{2}=-7.2780 \mathrm{dptr} ; \\
& \text { Radii: } r_{1}=+45.8275 \mathrm{~mm}, \quad r_{2}=+71.4479 \mathrm{~mm} ; \\
& \mathrm{A}_{1} \mathrm{H}=-3.9765 \mathrm{~mm}, \quad \mathrm{~A}_{2} \mathrm{H}^{\prime}=-6.1941 \mathrm{~mm}, \mathrm{~A}_{2} \mathrm{Z}^{\prime}=+26.2118 \mathrm{~mm} .
\end{aligned}
$$

The spherical aberration along the optical axis for the chief ray of slope $28^{\circ}$ is found to be $\mathrm{Z}^{\prime \prime} \mathrm{Z}^{\prime}=+0.7918 \mathrm{~mm}$, and the astigmatic difference on this ray: $\mathrm{P}^{\prime} \mathrm{Q}^{\prime}=-3$ i. $2294 \mathrm{~mm}$.

(3) For the following arbitrarily assigned values, namely:

$$
F=+6.0000 \text { dptr., } \mathrm{n}=1.52, d=3.0000 \mathrm{~mm}, \mathrm{~A}_{1} \mathrm{Z}=+36.5853 \mathrm{~mm} \text {; }
$$

the computation gives:

$$
\begin{aligned}
& F_{1}=+17.3130 \text { dptr., } F_{2}=-11.7133 \mathrm{dptr} ; \\
& \text { Radii: } r_{1}=+30.035 \mathrm{~mm}, r_{2}=+44.394 \mathrm{~mm} ; \\
& \mathrm{A}_{1} \mathrm{H}=-3.853 \mathrm{~mm}, \mathrm{~A}_{2} \mathrm{H}^{\prime}=-5.695 \mathrm{~mm}, \mathrm{~A}_{2} \mathrm{Z}^{\prime}=+26.849 \mathrm{~mm} .
\end{aligned}
$$

The spherical aberration along the optical axis for the chief ray of slope $28^{\circ}$ is found to be $\mathrm{Z}^{\prime \prime} \mathrm{Z}^{\prime}=-0.297 \mathrm{~mm}$, and the astigmatic difference on this ray: $\mathrm{P}^{\prime} \mathrm{Q}^{\prime}=-0.62 \mathrm{~mm}$.

(4) For the following arbitrarily assigned values, namely:

$$
F=+6.0000 \text { dptr., } \mathrm{n}=1.52 ; d=5.0605 \mathrm{~mm}, \mathrm{~A} Z \mathrm{Z}=+41.1523 \mathrm{~mm} \text {; }
$$

the computation gives:

$$
\begin{aligned}
& F_{1}=+14.0540 \mathrm{dptr}, F_{2}=-8.4493 \mathrm{dptr} ; \\
& \text { Radii: } r_{1}=+37.0002 \mathrm{~mm}, r_{2}=+61.5432 \mathrm{~mm} ; \\
& \mathrm{A}_{1} \mathrm{H}=-4.6884 \mathrm{~mm}, \mathrm{~A}_{2} \mathrm{H}^{\prime}=-7.7983 \mathrm{~mm}, \mathrm{~A}_{2} \mathrm{Z}^{\prime}=+28.1539 \mathrm{~mm} .
\end{aligned}
$$

The spherical aberration along the optical axis for the chief ray: of slope $28^{\circ}$ is found to be $Z^{\prime \prime} Z^{\prime}=-0.0412 \mathrm{~mm}$, and the astigmatic difference on this ray: $\mathrm{P}^{\prime} \mathrm{Q}^{\prime}=-2.701 \mathrm{~mm}$.

(5) For the following arbitrarily assigned values, namely:

$$
F=+6.0000 \text { dptr., } \mathrm{n}=1.52, d=6.1122 \mathrm{~mm} ; \mathrm{A}_{1} \mathrm{Z}=+41.6667 \mathrm{~mm} \text {; }
$$

the computation gives:

$$
\begin{aligned}
& F_{1}=+14.0540 \mathrm{dptr}, F_{2}=-8.5363 \mathrm{dptr} ; \\
& \text { Radii: } r_{1}=+37.0002 \mathrm{~mm}, r_{2}=+60.9165 \mathrm{~mm} ; \\
& \mathrm{A}_{1} \mathrm{H}=-5.7210 \mathrm{~mm}, \mathrm{~A}_{2} \mathrm{H}^{\prime}=-9.4190 \mathrm{~mm}^{\circ} \mathrm{A}_{2} \mathrm{Z}^{\prime}=+27.4779 \mathrm{~mm} ;
\end{aligned}
$$

The spherical aberration along the optical axis for the chief ray of slope $28^{\circ}$ is found to be $Z^{\prime \prime} Z^{\prime}=+0.7731 \mathrm{~mm}$, and the astigmatic difference on this ray: $\mathrm{P}^{\prime} \mathrm{Q}^{\prime}=-3.010 \mathrm{~mm}$.

(Note. As a matter of fact, numbers (4) and (5) above were computed by a different method from that explained under 
number (1); which amounted to assigning in advance the value of $F_{1}$ and calculating the corresponding value of $d$ by solving the quadratic equation in $\epsilon$, equation (5) above.)

(6) For the following arbitrarily assigned values, namely:

$F=-8.5000$ dptr., $\mathrm{n}=1.52, d=1.7647 \mathrm{~mm}, \mathrm{~A}_{1} Z=+23.5294 \mathrm{~mm}$;

the computation gives:

$F_{1}=+3.0627$ dptr., $F_{2}=-11.6038$ dptr.;

Radii: $r_{1}=+169.7828 \mathrm{~mm}, r_{2}=+44.8130 \mathrm{~mm}$;

$\mathrm{A}_{1} \mathrm{H}=+1.5849 . \mathrm{mm}, \mathrm{A}_{2} \mathrm{H}^{\prime}=+0.4183 \mathrm{~mm}, \mathrm{~A}_{2} \mathrm{Z}^{\prime}=+27.3945 \mathrm{~mm}$.

The spherical aberration along the optical axis for the chief ray of slope $28^{\circ}$ is found to be $\mathrm{Z}^{\prime \prime} \mathrm{Z}^{\prime}=-1.7592 \mathrm{~mm}$, and the astigmatic difference on this ray:

$$
\mathrm{P}^{\prime} \mathrm{Q}^{\prime}=+1.0746 \mathrm{~mm} \text {. }
$$

Lack of space prevents any discussion of these results, but it is hoped that they will be useful not only as illustrating the method of calculation but also as affording a basis of future calculation of lenses of this character.

\section{Curvatuke of the Stigmatic Image}

The so-called "Punktal" spectacle lens is a correction-glass which is designed to give a punctual image of the infinitely distant object-plane. The image of this plane in the lens is a curved surface which crosses the axis at the second focal point $F^{\prime}$ of the glass, which is likewise the position of the far point of the passive ametropic eye. The centre of curvature of this surface is a point on the axis which may be designated by $\mathrm{K}$, such that $\mathrm{F}^{\prime} \mathrm{K}=-\mathrm{n} f$, where $f=1 / F$ denotes the focal length of the lens (whose thickness is assumed "here to be negligible). The centre of the so-called "far point sphere" is at the centre of rotation of the eye, its radius being $\mathrm{F}^{\prime} \mathrm{Z}^{\prime}$. The image-surface is tangent to the far point sphere at $F^{\prime}$, but in general these two surfaces will not have any other point in common. The condition that the two surfaces shall coincide is that their centres $K$ and $Z^{\prime}$ shall be coincident; which means that we must have $f=-\mathrm{AZ}^{\prime} /(n-1)$, where $\mathrm{A}$ designates the optical centre of the infinitely thin lens. For $\mathrm{n}=1.52$ and $\mathrm{AZ}^{\prime}$ $=30 \mathrm{~mm}$, this condition gives $f=-57.7 \mathrm{~mm}$ or $F=-17.3 \mathrm{dptr}$. Accordingly, it may be concluded that the stigmatic image surface 
of a "Punktal" lens will coincide with the far-point sphere only for an unusually strong negative correction-glass. In general, therefore, the eye will have to make a little effort of accommodation in order to see distinctly in oblique directions through a "Punktal" correction-glass.

6. Merely as an additional note, it may be remarked here that the condition that an infinitely thin lens of refracting power $F$, placed in front of the eye at a distance $z^{\prime}=1 / Z^{\prime}$ from the centre of rotation of the eye, shall give a punctual or stigmatic image of an object lying in the primary focal plane of the lens (as in the case of an ordinary magnifying convex lens or of a spectacle lens for a very aged person who has lost entirely the power of accommodation) is as follows $\left(U=U_{1}=-F\right)$ :

$$
\left(\frac{\mathrm{n}}{\mathrm{n}-1} F-Z^{\prime}\right)^{2}+\frac{\mathrm{n}+2}{\mathrm{n}(\mathrm{n}-1)^{2}} F_{2}{ }^{2}-\frac{2 \mathrm{n}+1}{(\mathrm{n}-1)^{2}} F_{2} F+\frac{2(\mathrm{n}+1)}{\mathrm{n}(\mathrm{n}-1)} Z^{\prime} F_{2}=0 .
$$

Negative values of $F$ are excluded here, because if the object is in front of the eye, the lens must be positive. This equation also represents an ellipse which touches the $F$-axis at the point $F=(\mathrm{n}-1) Z^{\prime} / \mathrm{n}$. The maximum value of $F$ for $\mathrm{n}=1.52$ is $F_{\max }$ $=0.38158 Z^{\prime}=12.72 \mathrm{dptr}$. for $Z^{\prime}=33 \frac{1}{3} \mathrm{~d} \mathrm{dptr}$.

Department of Physics,

Columbia University, New York, N. Y.

July 9, 1921. 